\title{
Optical Performance as a Function Dynamic Mechanical Loading
}

\author{
Victor L. Genberg*, Keith B. Doyle, Gregory J. Michels \\ Sigmadyne, Inc. \\ 803 West Ave, Rochester, NY
}

\begin{abstract}
Mechanical vibrations have a large impact on the optical performance of many systems. In order to accurately predict optical performance, an integrated optomechanical analysis is required. It is difficult to pass finite element vibration data to optical programs since harmonic response includes phase angles due to damping, and random response has lost all phase information.
\end{abstract}

This paper discusses a new technique to decompose harmonic and random response into pointing, focus, and wavefront error components in an efficient manner. This technique is compatible with commonly used error budgets. A method to identify key contributors to each error component is also presented.

Keywords: optomechanical analysis, integrated modeling, vibrations, jitter, Zernike polynomials

\subsection{JITTER}

\subsection{Image Motion Equations}

Line-of-sight (LOS) jitter is the apparent motion of a stationary object as viewed by an optical sensor. Image motion is often defined as the motion of the "gut ray" on the image plane (Figure 1).
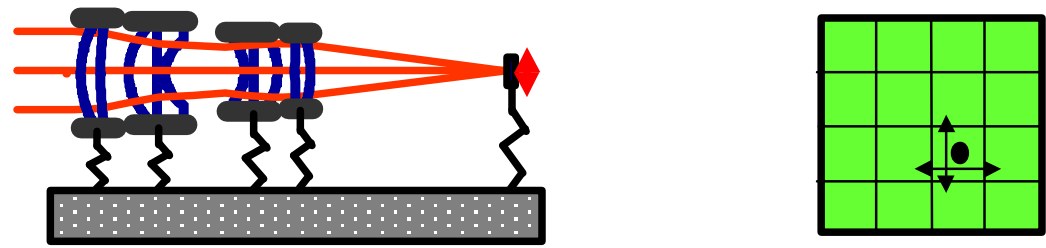

Figure 1: Image Motion

In an optical system with small motions, a linear equation using optical sensitivity coefficients [L] for rigid body motions $[\mathrm{T}]$ of each optical surface ${ }^{1}$. These sensitivities are typically obtained from an optical design code.

$$
\left\{\begin{array}{c}
\Delta_{x} \\
\Delta_{y}
\end{array}\right\}=[L]_{i m g}[T]_{\text {optics }}
$$

\subsection{Image Motion in FE Model}

The image motion equations can be entered directly into most finite element (FE) programs. For example, in Nastran linear equations may be entered as MPC data. Care must be taken to insure that coordinate systems, sign conventions, and units are transformed from the optical code correctly. It is common for optical codes to use left-handed rotations in degrees, while FE data must be in right-handed rotations in radians.

If the image motion equations are entered into the FE model, system level dynamic jitter may be output directly from a dynamic response analysis in the FE program as a time history plot in transient, a frequency response plot in harmonic, or a PSD plot in random response analysis.

*genberg@sigmadyne.com; phone (585) 235-7460 


\subsection{Flexible Optics}

If an optic moves as a rigid body, then a single node in the FE model can represent each the optical surface motion. For example, in an optical bench with many small optics, the model could look like Figure 2:

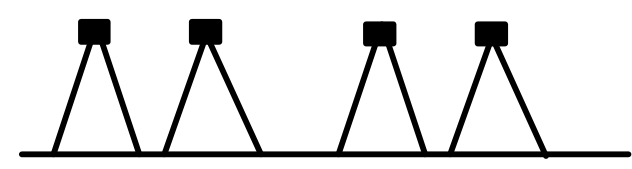

Figure 2: Lumped optics

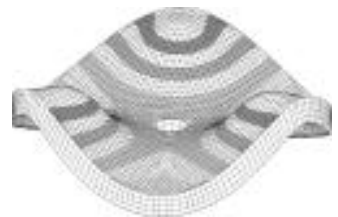

Figure 3: Flexible optics

For flexible optics, such as large primary mirrors (Figure 3), the "average" rigid body motion can be calculated by interpolation elements (RBE3 in Nastran) or from linear equations as follows. Let the best-fit rigid-body motions of a surface be defined as a vector [T] of 6 terms:

$$
\begin{aligned}
& T_{x}, T_{y}, T_{z}=\text { translations along coordinate axes }\left(T_{x}, T_{y}=\text { decenters, } T_{z}=\text { bias }\right) \\
& \left.R_{x}, R_{y}, R_{z}=\text { rotations about coordinate axes (right-hand rule }=+\right)\left(R_{x}, R_{y}=\text { tilts }\right)
\end{aligned}
$$

Then the displacement of node i due to these motions is:

$$
\begin{aligned}
& d \tilde{x}_{i}=T_{x}+z_{i} R_{y}-y_{i} R_{z} \\
& d \tilde{y}_{i}=T_{y}-z_{i} R_{x}+x_{i} R_{z} \\
& d \tilde{z}_{i}=T_{z}+y_{i} R_{x}-x_{i} R_{y}
\end{aligned}
$$

Define the error $(E)$ as the area-weighted (w) difference between the actual nodal displacements $\left(\mathrm{dx}_{\mathrm{i}}\right)$ and the rigid-body values calculated above.

$$
E=\sum_{i} w_{i}\left(d x_{i}-d \tilde{x}_{i}\right)^{2}+w_{i}\left(d y_{i}-d \tilde{y}_{i}\right)^{2}+w_{i}\left(d z_{i}^{\prime}-d \tilde{z}_{i}\right)^{2}
$$

To find the best-fit motions $\left(T_{x}, T_{y}, T_{z}, R_{x}, R_{y}, R_{z}\right)$, minimize $E$, by taking partial derivatives with respect to each term and setting the result to zero. A typical equation is:

$$
\frac{\partial E}{\partial T_{x}}=0
$$

which leads to:

$$
\sum_{i} w_{i} T_{x}+\sum_{i} w_{i} z_{i} R_{y}-\sum_{i} w_{i} y_{i} R_{z}=\sum_{i} w_{i} d x_{i}
$$

After setting all 6 derivatives to zero, there are 6 simultaneous equations to solve. Note that the axial corrected surface displacements $^{2}\left(\mathrm{dz}^{\prime}\right)$ is used in these calculations.

\subsection{COMPONENT DISTORTION}

\subsection{Decomposition}

When flexible optics distort, the distortion can be decomposed into average rigid body motion [T], power $\{\mathrm{P}\}$, and left over residual surface error $\{\mathrm{V}\}$. If the decomposition is conducted on the undamped, dynamic mode shapes (eigenvectors) then all data is real (not complex), simplifying the mathematics. 


\subsection{Harmonic and Random Response}

Modal analysis techniques, commonly used for dynamic response, assume that the physical response is a linear combination of the modal responses. In steady state harmonic response, the applied load is $F=\mathrm{F} \mathrm{e}^{\mathrm{i} \omega t}$ and the resulting displacement is $U=\mathrm{U} \mathrm{e}^{\mathrm{i} \omega t}$. The harmonic equation is

$$
[M]\{\ddot{U}\}+[B]\{\dot{U}\}+[K]\{U\}=\{F\}
$$

In the modal approach,

$$
\{U\}_{i}=\sum_{m}\{\phi\}_{m} \zeta_{m i}=[\Phi]\{Q\}_{i}
$$

where $\zeta_{\mathrm{mi}}$ is the modal participation factor for mode $\mathrm{m}$ at load step i. Substituting the assumed harmonic form of the displacement vector and Equation (6) into the response Equation (5), canceling $\mathrm{e}^{\mathrm{i} \omega t}$ and premultiplying by $\Phi^{\mathrm{T}}$ gives,

$$
[\Phi]^{T}[M][\Phi]\{\ddot{Q}\}_{i}+[\Phi]^{T}[B][\Phi]\{\dot{Q}\}_{i}+[\Phi]^{T}[K][\Phi]\{Q\}_{i}=[\Phi]^{T}\{F\}_{i}
$$

which is a series of uncoupled 1 DOF equations (assuming modal damping) to solve. These uncoupled equations can be written in the form,

$$
m \check{\zeta}_{m i}+b \zeta_{m i}+k \zeta_{m i}=f_{m i},
$$

where $\mathrm{m}, \mathrm{b}, \mathrm{k}, \mathrm{f}$ are modal mass, modal damping, modal stiffness and modal force, respectively. The solution to Equation (8) is

$$
\zeta_{\mathrm{mi}}=\mathrm{f}_{\mathrm{mi}} /\left[\left(\mathrm{k}-\mathrm{m} \omega^{2}\right)+\mathrm{ib} \omega\right]
$$

where $\mathrm{b}=2 \mathrm{c} / \mathrm{c}_{\mathrm{c}}$. Note that $\zeta$ is complex containing both magnitude and phase. Any response quantity such as displacement $\{U\}$ can be found from the modal displacements $\{\phi\}$ by eqn (6).

For optical performance calculation, efficiencies can be realized using this modal approach. For each surface, mode $\mathrm{j}$ $\left(\Phi_{\mathrm{j}}\right)$ is decomposed into,

$$
\begin{aligned}
& T_{j}=\text { pointing vector for the } j \text { th mode } \\
& P_{j}=\text { power for the } j \text { th mode } \\
& V_{j}=\text { the residual vector for the } j \text { th mode }=\Phi_{j}-T_{j}-P_{j}
\end{aligned}
$$

then, the net surface pointing error and the net surface power for each surface are obtained from,

$$
T_{n}=\sum \varsigma_{j} T_{j} \quad P_{n}=\sum \varsigma_{j} P_{j}
$$

and the net surface RMS is obtained from the combined residual vectors,

$$
R M S_{n}=R M S\left(\sum \varsigma_{j} V_{j}\right)
$$

These techniques have been available in $\mathrm{SigFit}^{3}$ for some time. The advantage over conducting the dynamic response within an FE code is that the FE results cannot be decomposed easily. In harmonic response, the FE results would provide magnitude and phase information on each surface node. Pointing error calculations must account for the 
difference in phase for each node. If a random response is calculated, then all phase information about each node is lost and pointing error (R-B motion in Figure 4) cannot be distinguished from surface distortion (surface RMS in Figure 5).

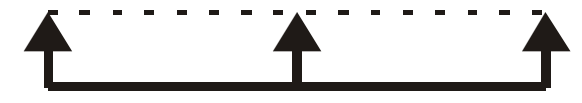

Figure 4: All RB motion, No elastic distortion

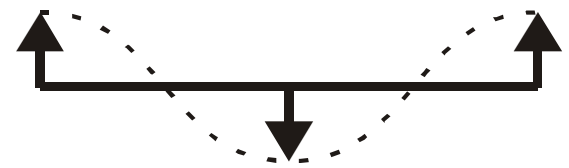

Figure 5: No RB motion, All elastic distortion

The above rigid-body decomposition technique ${ }^{4}$ allows meaningful pointing and surface distortion calculations, even in random response. As programmed in SigFit, the technique allows for identification of each modes contribution so that design improvements can be made efficiently. The limitation is that the distortion of each surface is a net surface RMS effect that must be RSS'd to get a system response. The method in the next section allows more detailed prediction of system response.

\subsection{INTEGRATED DYNAMIC RESPONSE}

\subsection{Notation}

$\mathrm{N}=$ Total number of optical surfaces in model

$\mathrm{T}=$ Total number of Rigid-Body (R-B) and Zernike terms fit to each optical surface

$\mathrm{M}=$ Total number of dynamic modes used

$\mathrm{i}$ = Load step number (either static load case, frequency step, or time step)

$\mathrm{S}_{\mathrm{kt}}{ }^{\mathrm{n}}=$ Sensitivity matrix $=$ Zernike $(\mathrm{k})$ at exit pupil for each R-B / Zernike (t) at surface (n)

$\mathrm{D}_{\mathrm{ti}}{ }^{\mathrm{n}}=$ Static displacement decomposition $=$ fit each deformation case (i) with R-B /Zernike (t) at surface (n)

$\mathrm{C}_{\mathrm{tm}}{ }^{\mathrm{n}}=$ Mode decomposition $=$ fit each mode $(\mathrm{m})$ with R-B /Zernike ( $\mathrm{t}$ ) at surface (n)

$\mathrm{Z}_{\mathrm{ki}}^{0}=$ Zernikes (k) at output location (0) for load step (i)

\subsection{System Wavefront}

Zernike polynomials $s^{5}$ may be fit to any individual surface deformation ${ }^{6}$. If each optical surface (n) in a static deformed load case (i) are decomposed into rigid-body motions and Zernike coefficients $\mathrm{D}_{\mathrm{ti}}{ }^{\mathrm{n}}$, then each Zernike (k) at the exit pupil (0) can be approximated by a linear combination ${ }^{7}$

$$
Z_{k i}{ }^{0}=\sum_{n}^{N} \sum_{t}^{T} S_{k t}^{n} D_{t i}^{n}
$$

The sensitivity matrix $\mathrm{S}$ is obtained from an optical design program. In our work, a script was used to produce the large $\mathrm{S}$ matrix from Code V. A surface fitting program such as SigFit will calculate the D matrix for each load case. SigFit has been modified to read the $\mathrm{S}$ matrix and calculate and report $\mathrm{Z}$ along with the associated wavefront RMS, P-V and contour plots. This approximation in equation (12) is valid for linear systems in which the individual surface deformations are accurately described by Zernike coefficients.

\subsection{System Dynamic Response}

For optical performance under dynamic loading,, efficiencies can be realized using the modal approach. If each mode $\Phi_{\mathrm{m}}$ is decomposed into R-B and Zernike coefficients $\mathrm{C}_{\mathrm{jm}}{ }^{\mathrm{n}}$, the net dynamic response is the sum of modal contributions:

$$
Z_{k i}{ }^{0}=\sum_{n}^{N} \sum_{t}^{T} \sum_{m}^{M} S_{k t}{ }^{n} C_{t m}{ }^{n} \zeta_{m i}
$$


The above calculations may be done in any order. After the Zernike fit of each mode shape, one could multiply and sum over surfaces (n) to obtain a modal result at the exit pupil:

$$
A_{k m}{ }^{0}=\sum_{n}^{N} \sum_{t}^{T} S_{k t}{ }^{n} C_{t m}{ }^{n}
$$

Then during the modal response analysis, each Zernike $(\mathrm{k})$ at the exit pupil for load step (i) could be found more efficiently by:

$$
Z_{k i}{ }^{0}=\sum_{m}^{M} A_{k m}{ }^{0} \zeta_{m i}
$$

Each Zernike coefficient may be plotted verses time (transient analysis) or verses frequency (harmonic analysis). In a random response analysis, the PSD of each Zernike coefficient may be output or the integrated 1-sigma response reported. In the SigFit implementation, each modes contribution to the net surface RMS is reported, so major modal contributors can be identified. SigFit also outputs a net wavefront map for selected times or frequencies in a nodal file format compatible with the FE graphics code allowing wavefront contours at the exit pupil.

\subsection{ADVANTAGES}

\subsection{Conventional Design Process}

In the conventional opto-mechanical design process, the optical design is completed first. Next, the mechanical engineer must cycle through designs to meet optical performance requirements. However, to evaluate a design, the mechanical engineer must pass the mechanical response to the optical engineer for evaluation as shown in Figure 6. The overall process is time-consuming and prone to error.

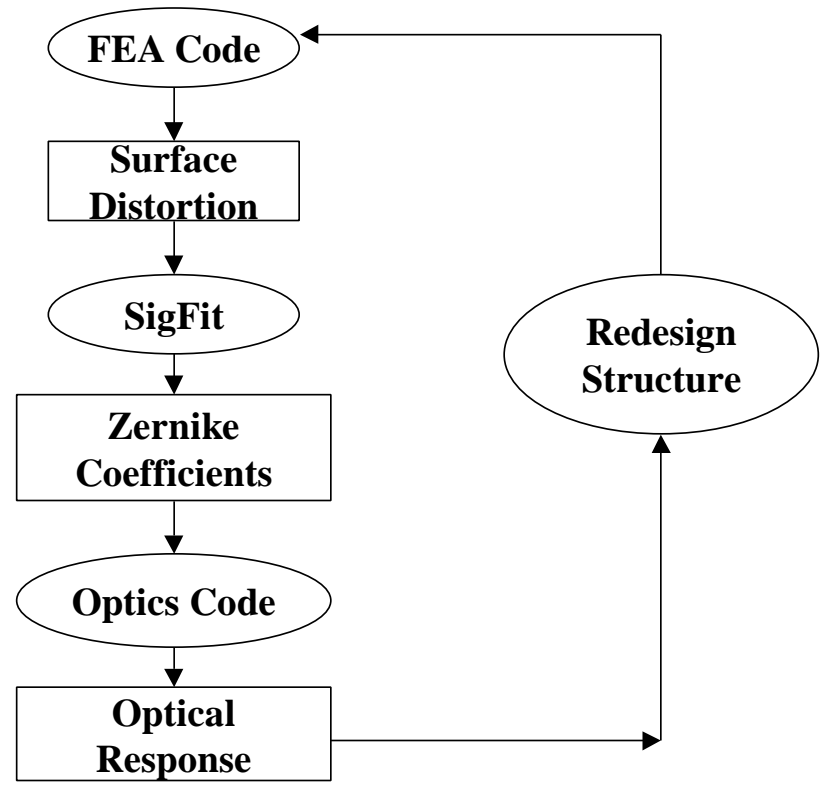

Figure 6: Conventional Opto-mechanical Design Process 


\subsection{New Design Process}

With the new system level response capability ${ }^{8}$ in SigFit, the design process can be streamlined. When the optical design is complete, the optical engineer runs a sensitivity analysis to obtain the sensitivity matrix. The mechanical engineer can then cycle through the mechanical design process (Figure 7) and make design decisions based on optical performance, without the conventional passing of data back and forth.

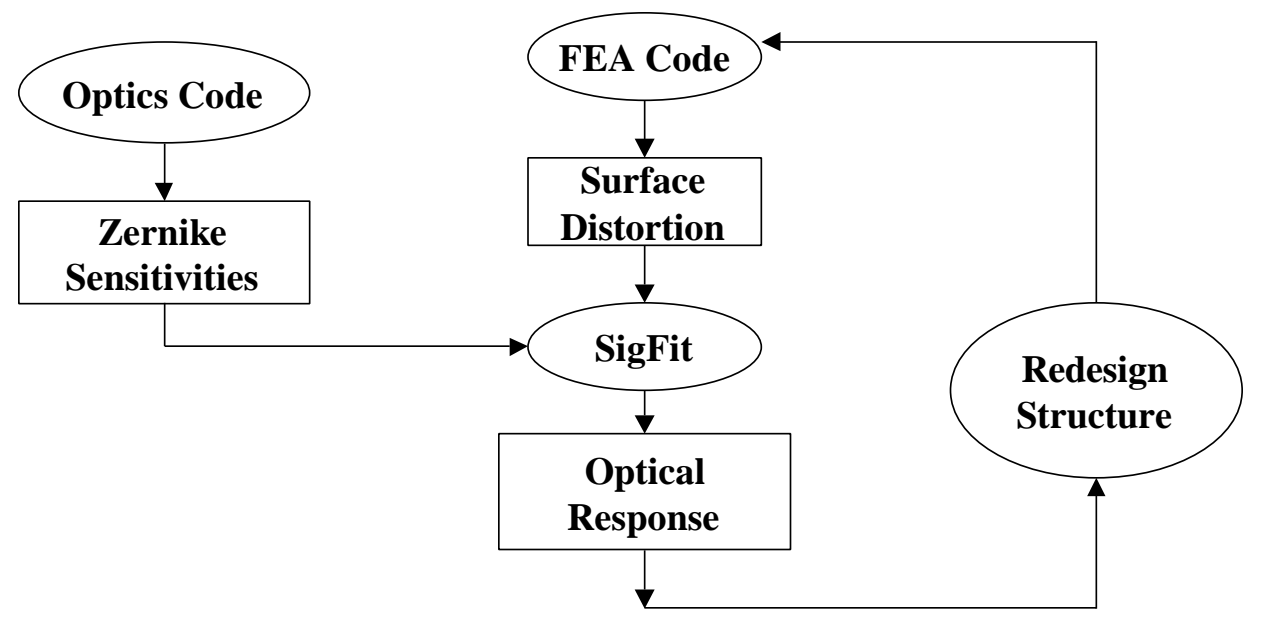

Figure 7: New Opto-mechanical Design Process

\subsection{SUMMARY}

Techniques and tools have been discussed for calculating optical performance as a function of dynamic loading. The new technique using optical sensitivities offers new efficiencies in the opto-mechanical design process.

\subsection{REFERENCES}

1) K. B. Doyle, V. L. Genberg, G. J. Michels, Integrated Optomechanical Analysis, SPIE Tutorial Text 58, 2002.

2) V. L. Genberg, G. J. Michels, K. B. Doyle,"Making Mechanical FEA Results Useful in Optical Design", SPIE Paper 4769-04, 2002.

3) V. L. Genberg, SigFit Reference Manual, Sigmadyne, Inc., Rochester, NY, 2003.

4) V. L. Genberg, G. J. Michels, "Opto-mechanical analysis of segmented/adaptive optics," SPIE Paper 4444-10, 2001.

5) V. L. Genberg, G. J. Michels, K. B. Doyle, “Orthogonality of Zernike Polynomials,", SPIE Paper 4771-33, 2002.

6) V. L. Genberg, “Optical Surface Evaluation”, SPIE Paper 450-08, 1983.

7) K. B. Doyle, V. L. Genberg, G. J. Michels, " Integrated optomechanical analysis of adaptive optical systems", SPIE Paper 5178-5, 2003.

8) V. L. Genberg, K. B. Doyle, G. J. Michels, "Optomechanical Design and Analysis of Adaptive Optical Systems using FEA and Optical Design Software", FEMCI Workshop, NASA GSFC, May 2003. 